\title{
Power Control in WLANs for Optimization of Social Fairness
}

\author{
Vaggelis G. Douros, Pantelis A. Frangoudis, Konstantinos Katsaros and George C. Polyzos
}

\begin{abstract}
Wide deployment of Wireless Local Area Networks (WLANs), especially in modern densely-populated metropolitan areas, constitutes a leap towards ubiquitous wireless network access. This is largely attributable to their low installation and operational cost and mainly their operation in unlicensed bands. Together with the clear benefits of increased WLAN coverage though, new challenges have emerged; dense WLAN deployments lead to significant interference among neighbor networks, making its mitigation a matter of crucial importance for their performance. To this end, transmission power control is a natural choice to limit interference. In this paper, we propose and evaluate two algorithms (FirstMax and BestMax) for WLAN Access Points (APs) to cooperatively control their transmission power levels, leading to more fair and more efficient spectrum usage. Simulations demonstrate that the proposed algorithms (and especially BestMax) are far more profitable and fair choices than transmitting at the maximum feasible power. Consequently, an optimization of the social fairness can be achieved.
\end{abstract}

Index Terms-Interference Mitigation, Power Control, Unlicensed Spectrum, Wireless Local Area Networks.

\section{INTRODUCTION}

$\mathrm{T}$ HE development of wireless communications and networks is far more visible than ever. A representative example of this evolution is the establishment of a multitude of Wireless Local Area Networks (WLANs) that appear in various locations (buildings, university campuses, hotels), serving many users. However, the increasing demand for wireless and often mobile services leads to the need for an efficient usage of the spectrum. Consequently, spectrum sharing is an issue of crucial importance, considering that it is a finite resource. Spectrum is split into licensed and unlicensed bands. The former are characterized by exclusive usage rights of each band for the owners of the license. On the other hand, access to unlicensed bands is open to all and free. This facilitates the deployment of technologies operating in such frequencies and explains the boom in the usage of the IEEE 802.11 standards family for WLANs, also known as $W i-F i$, as well as the IEEE 802.15 standards for shorter range communications (Bluetooth, ZigBee).

The IEEE 802.11 standard specifies that the available spectrum is divided in channels. In its 11.b and 11.g variants, only 3 of these channels are non-overlapping. This implies that up to 3 WLANs can coexist in an area and operate on

The authors are with the Mobile Multimedia Laboratory, Department of Informatics, Athens University of Economics and Business, 10434 Athens, Greece (e-mails: douros06@aueb.gr, pfrag@aueb.gr, ntinos@aueb.gr, polyzos@aueb.gr). non-overlapping channels. More than 3 IEEE $802.11 \mathrm{~b} / \mathrm{g}$ Access Points (APs) in the same area means that some of them are assigned (at least partially) overlapping channels and thus suffer from interference, which in turn results in degraded performance.

Combating interference has emerged as a significant research issue and a number of power control approaches for interference mitigation $[1,2]$ have been proposed. The model that we assume includes a number of APs that transmit using the same channel and try to adjust their transmission power for the optimization of a network performance metric, such as the minimization of the user-perceived interference, or the maximization of the signal to interference plus noise ratio (SINR) of their users. These metrics are closely related to an AP's income, which is an expression of an AP's profit or utility, and is a function of the network performance achieved by the AP's clients, as well as their number (that can be supported at that performance level).

To maximize the income, as defined above, an obvious short-sighted choice would be to transmit at the maximum permitted power. This has the advantage that APs cover the maximum geographical area, so they can serve as many users as possible. On the other hand, maximizing transmission power implies that the operational expenses of the APs are highest, while interference among neighbor APs is also at its maximum.

The contribution of this paper is the design and evaluation of two algorithms which, assuming that all APs cooperate to reduce their transmission power simultaneously and to the same level, lead to a fair distribution of the APs' incomes so as to achieve an optimization of the social fairness, i.e. a fair sharing of a common good (the spectrum).

The remainder of this paper is organized as follows. Related work is presented in Section II. Our algorithms are presented in detail in Section III and a set of simulations which evaluate their performance are given in Section IV. Concluding remarks and discussion of future work are provided in Section V.

\section{RELATED WORK}

Among others, Akella et al. [10] have observed the severe interference problems that appear in present-day chaotic WLAN deployments and have proposed mechanisms based on power control and transmission rate adaptation to tackle them. With a similar motivation, and mainly for the case of high density enterprise or campus-wide WLANs, the authors in [1] present optimization methods to limit interference through power control. More specifically, they use a Gibbs sampler to 
approximate a globally optimal solution through local optimizations.

There are many papers which study power control with game theory. In [2], cooperative game theory is used so as to jointly tackle the problem of channel selection and power control in cognitive radio networks [5]. Players are cognitive radios and their utility functions are defined by taking into account the sum of the interference that is experienced by their overlapping APs.

Authors in [4] consider that players can concurrently transmit in more than one channel and their utility functions depend on the receiving SNR. Besides cooperative game theory, there are occasions where non-cooperative game theory has been applied with success in WLANs. In [3], the authors propose various strategies (mainly tit-for-tat) which can be used in a 2-player game so as to maximize the utility functions of the players.

Finally, some recent papers deal with the application of the cell breathing technique in WLANs, which led to very interesting results in cellular networks [7]. The ultimate goal combines the concept of cell breathing along with power control in order to develop load balancing algorithms. These algorithms should be used in cases where sporadic congestion of users appears in a WLAN [6].

\section{PROPOSED AlgorithMS}

\section{A. Prerequisites}

We assume that there exist in the same area $M$ APs, which can serve $N$ users, who may be located in the same geographical area. All APs use the same channel (as those are available from the IEEE $802.11 \mathrm{~b} / \mathrm{g}$ ), which means that users within the transmission range of two or more APs suffer from interference. We also suppose that, initially, each AP transmits with maximum possible power $\left(P_{\max }\right)$, so as to be able to cover the maximum number of users (even the furthermost ones accessible by the AP). Moreover, we assume that the transmission power levels are discrete, which holds for the APs of several manufacturers. All APs simultaneously reduce their transmission power by a constant quantity. We also consider that all APs are fully synchronized (this can be easily implemented in practice through the exchange of a signaling message through the Internet-to a significant accuracy). Finally, we assume that each AP belongs to a different owner.

Our approaches are AP-centric, in the sense that we are interested in studying under which circumstances should incomes of all APs correspond to as fair a distribution as possible. This implies that our mechanisms lead to states where the percentage of losses of maximum feasible incomes of APs should be allocated in the fairest manner. It is crucial to observe that the above goals could only be achieved provided that all APs follow exactly the proposed schemes.

\section{B. Income Computation}

Let us suppose that each of the $N$ users of the topology has been connected with one of the $M$ APs, which initially transmit at maximum power.

In the new state, i.e. after the reduction of transmission power, it is possible that some changes concerning the userAP associations, will take place. First, a user may not belong to the new coverage area of the AP that was previously attached to. This means that either he/she will try to connect with another AP (if that is possible), or he/she will not be able to be served by any of the available APs. Second, the reduction of the transmission power for all APs implies the shrinkage of the overlapping coverage area for the APs. Consequently, a user who previously suffered interference by some APs, may now suffer from interference from fewer or, none.

In the new state and after the above mentioned changes for the users, APs must compute their incomes and compare them with those of the previous state. In other words, they have to add the incomes of all users they serve (as reported to them by the clients). We define the function $C_{i, k}$ that gives the income from user $i$ who is attached to AP $k$ as proportional to the quantity $u_{i, k}(d)$, which is defined by eq. (1).

$u_{i, k}(d)=\left\{\begin{array}{l}\frac{c}{d_{i, k}}, i \text { does not feel interference } \\ \frac{c}{d_{i, k}}-\sum_{j \neq k} \frac{c}{d_{i, j}}, \text { ifeels interference }\end{array}\right\}$

where $c$ is a constant and the quantity $d_{i, j}(2)$ expresses the Euclidian distance between a user $i$ and an $\mathrm{AP} j$ with which it is either attached or belongs to its coverage area.

$d_{i, j}=\left\{\begin{array}{l}\infty, \mathrm{i} \notin \text { coverage of } \mathrm{AP} \mathrm{j} \\ \sqrt{\left(x_{i}-x_{j}\right)^{2}+\left(y_{i}-y_{j}\right)^{2}}, i \in \text { coverage of AP } \mathrm{j}\end{array}\right\}$

Equations (1) and (2) correspond to the inverse proportional correlation of the income of an AP with the total signal-tointerference-plus-noise-ratio (SINR) that its users receive.

So, the goal for each AP $k$, is the computation of the quantity $C_{k}$ which is defined as

$$
C_{k}=\sum_{i=1}^{N_{k}} C_{i, k}
$$

where the upper limit $N_{k}$ is the number of users that have been attached to AP $k$.

\section{Algorithm FirstMax}

When each AP computes its total income -using eq. (1), (2) and (3), it compares that with the one that corresponds to the previous transmission power. If in the new state, the income for each AP is bigger (or at least equal, as in that case there is at least the reduction in the expenses of the AP because of the lower power level) than the income in the previous state, then the power decrease was a wise choice and the simultaneous reduction of the APs' power continues. The first time that an AP's income deteriorates, the whole process stops and all APs select the exact previous power state as the final choice (to transmit at). 


\section{Algorithm BestMax}

BestMax aims at finding a fairer distribution of AP's incomes. It uses a slight but significant variation of the previous algorithm: APs simultaneously reduce their transmission power until the point where it is impossible to improve the income of at least one AP. This state corresponds to the transmission power level where no user feels interference from an AP. Taking into account the definition of an AP's income, it is easy to prove that further reduction of the transmission power cannot lead to an AP's income increase.

Based on the above, we propose the following process:

a) Find the value of the transmission power that maximizes the income of an AP independently of the income of other APs.

b) Repeat the previous step for all APs of the topology.

c) Find the value of the transmission power that the sum of the percentage losses from the maximum feasible incomes of each AP is minimized. The use of percentage losses (instead of absolute losses) is justified due to our assumption that each AP belongs to a different owner.

\section{E. Comparison of Incomes of APs after the Application of First Max and Best Max}

Fig. 1 shows the variation of the income of the participating APs along with the transmission power for an instance of the two algorithms execution with $\mathrm{M}=2$. We have pointed out values of transmission power which correspond to the application of FirstMax and BestMax. As we notice, FirstMax terminates at a very early point, as this was the first local maximum for the income of an AP. This was a rather bad choice for the transmission power for each AP, as there are many lower values of the power for which the incomes for both APs are quite larger.

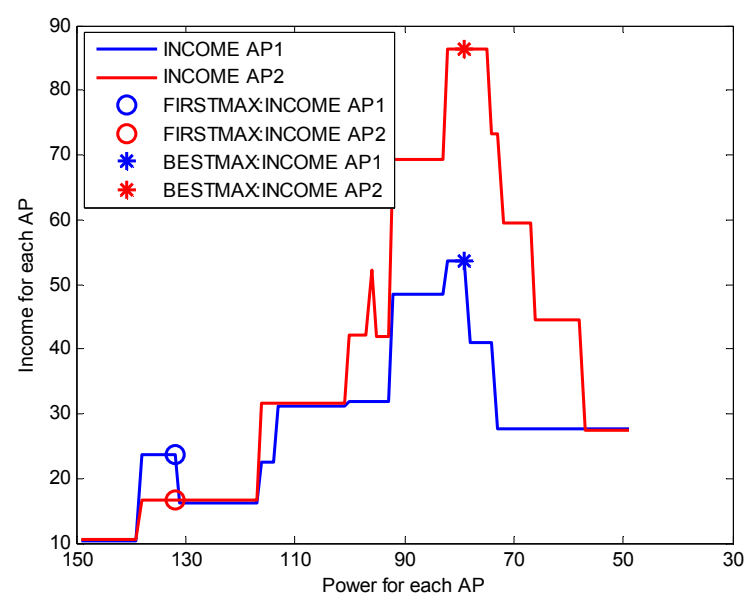

Fig. 1. Variation of the income for $\mathrm{AP}_{1}$ and $\mathrm{AP}_{2}$ along with their transmission power.
On the other hand, in this particular case, the application of the BestMax algorithm is far more beneficial for both APs, as it adjusts their transmission power to a point where incomes for both APs are (globally) maximized. Even in the general case (where the maximization of incomes for each AP does not correspond into the same transmission power), BestMax finds a better/fairer maximum than FirstMax and leads to a fairer distribution of incomes of all APs of the topology.

Figures 2 to 4 depict incomes during the transmission of all APs at maximum power, after the application of FirstMax and after the application of BestMax respectively, in an example topology. Comparing Fig. 2 to Fig. 3, we observe that the only difference is that the radius coverage $R$ after the application of FirstMax is shrunk at $146 \mathrm{~m}$ (the initial one was $150 \mathrm{~m}$ ). That means that FirstMax leads to the same distribution of incomes for both APs (the only plus has to do with the partial reduction of their expenses due to the reduction of the transmission power).

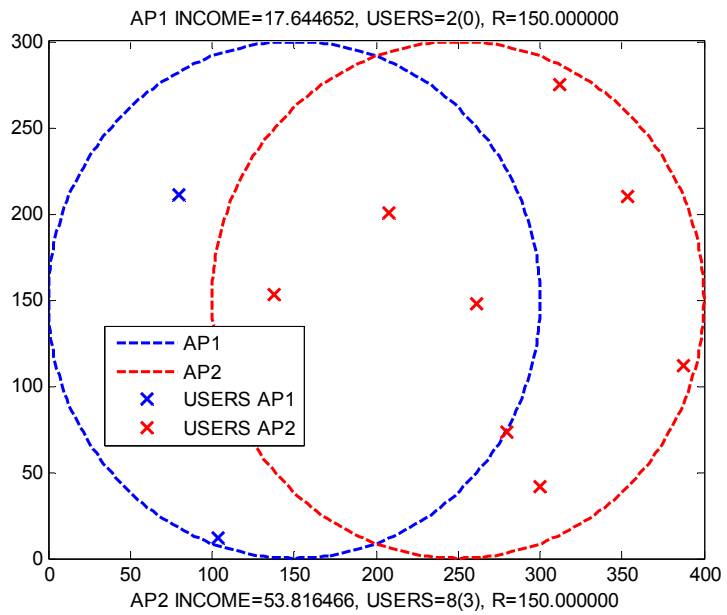

Fig. 2. Algorithm Pmax: Positions of users, number of users-total and interfered- for each AP, income of each AP, coverage radius.

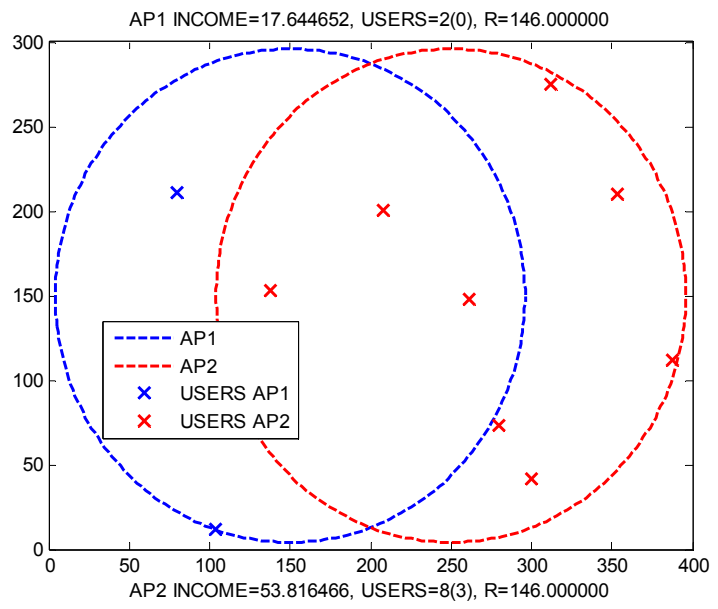

Fig. 3. Algorithm FirstMax: Positions of users, number of users-total and interfered- for each AP, income of each AP, coverage radius. 


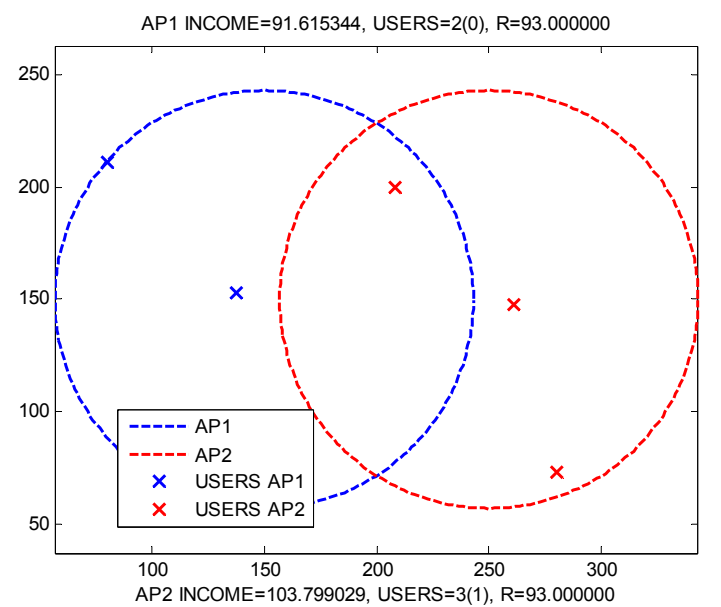

Fig. 4. Algorithm BestMax: Positions of users, number of users-total and interfered-for each AP, income of each AP, coverage radius.

Fig. 4 depicts positions of users and incomes of APs after the application of BestMax. We notice that $\mathrm{AP}_{1}$ 's income increases from 17 units to 91 units, while $\mathrm{AP}_{2}$ 's income from 53 units to 103 units. Moreover, this topology contains only 5 users, whereas the previous ones contained 10 users. We are wondering whether there exists an explanation for the "paradox": a decrease in the number of users leads to an increase of the income for both APs!

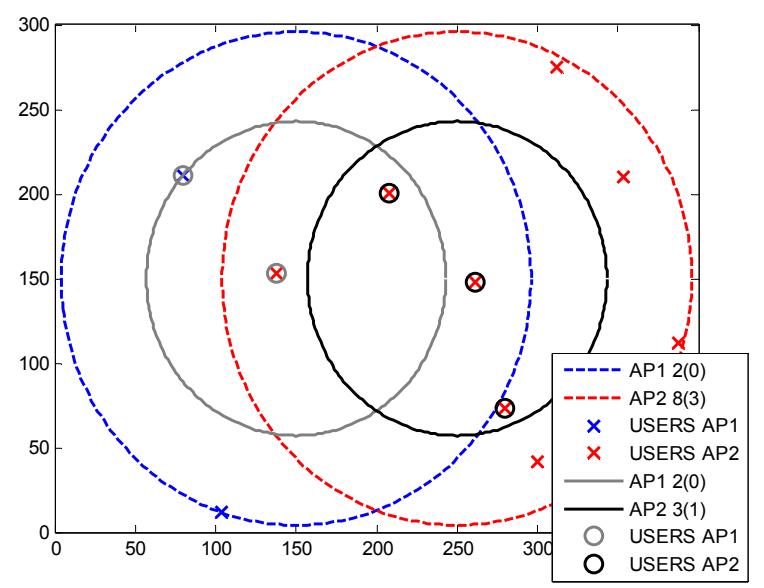

Fig. 5. Algorithms FirstMax and BestMax on the same diagram.

A nice interpretation of the above mentioned question arises if we present algorithms FirstMax and BestMax on the same diagram (Fig. 5). The two big circles (colored blue and red) correspond to $\mathrm{AP}_{1} \mathrm{Kall}_{\mathrm{AP}}$ after the application of FirstMax. Their users are represented with the same colors too. Similarly, but with colors grey and black, $\mathrm{AP}_{1}, \mathrm{AP}_{2}$ and their corresponding users are depicted after the application of BestMax.

Concerning $\mathrm{AP}_{1}$, we notice that BestMax differs from FirstMax to just one user. This new user is found very near to the center of $\mathrm{AP}_{1}$ and he/she does not experience interference from $\mathrm{AP}_{2}$ (black circle). Consequently, this user is far more profitable from the user that was marginally served by $\mathrm{AP}_{1}$, after the application of FirstMax (blue circle, left down user).
Concerning $\mathrm{AP}_{2}$, we observe that BestMax differs from FirstMax in five users. These users are represented with red symbol ' $x$ ', without being represented with a black ' $o$ '. We can notice that these users were found quite far from the center of $\mathrm{AP}_{2}$, without offering great income to it, due to interference -consider eq. (1) and (2). On the other hand, the benefit that arises for $\mathrm{AP}_{2}$ after the application of BestMax is that its two users most to the right do not suffer anymore interference from $\mathrm{AP}_{1}$ (grey circle). Therefore, the fact that $\mathrm{AP}_{2}$ can now serve two users that are found near it and they do not suffer interference is far more beneficial than serving five users that were located far from it without suffering interference, but their two nearest users felt interference (FirstMax application).

In conclusion, it is clear that the target goal for the APs should be to serve in an efficient way (i.e. with little or no interference) users that are located near them, instead of trying to serve as many users as possible, with no regard to interference. Moreover, it is obvious that the owners of the APs should not fear from a temporary decrease of their expenses during the reduction of the transmission's power. It is proven that this reduction can be very profitable for them as the adoption of BestMax may increase their incomes significantly.

\section{Performance Evaluation}

We have simulated topologies - using $\mathrm{C}$ and Matlab- with: (i) two APs $(\mathrm{M}=2)$ and (ii) three APs $(\mathrm{M}=3)$ that form an equilateral triangle. The number of users was 10, 30, 50 and 100 respectively, uniformly distributed in the simulated area. For all combinations of numbers of APs and users, we executed 10000 simulations. User mobility was assumed limited, as users were assumed to follow the quasi-static model, i.e. they could move throughout the geographical area but slowly and were expected to stay in the same place for a long time [8]. This assumption is in agreement with previous studies concerning user mobility in WLANs [9]. Moreover, we suppose that all users appear at the same time in the topology and no arrivals or voluntary departures take place during the simulations. In other words, all users are served as long as they belong to the coverage area of at least one AP. Finally, the value of the constant $c$ defined in eq. (1) was set to 1000 (a suitable value for normalization of incomes of APs).

Figs. 6 and 7 help us compare the average percentage income improvements that arise after the application of either FirstMax or BestMax, instead of transmission of all APs at the maximum possible power. We observe that when the topology consists of 2 APs, BestMax outperforms Pmax, as the former leads to an average increase from $300 \%$ to $500 \%$ (depending on the number of users of the topology). On the other hand, the average percentage improvement per AP after the application of FirstMax is smaller and varies from $10 \%$ to $70 \%$. We also notice that this improvement is remarkable only when the total number of users is 10 (in the example). 
Correspondingly, when the topology consists of 3 APs, BestMax leads to even larger incomes than Pmax, as the average percentage increase fluctuates from $500 \%$ to $1400 \%$ ! On the other hand, results of the application of FirstMax remain the same with the ones of the previous figure. If we compare these two diagrams, we can easily notice that BestMax is by far the best algorithm to choose. This result is completely predictable, according to the analysis of the previous sections.

Finally, we would like to mention that the performance of BestMax is characterized by a high probability that the incomes of all APs would increase by at least $100 \%$. These conclusions, that arose after a statistical analysis of the results of the simulations (we do not present them here in detail due to space constraints), apply to every considered combination of numbers of APs and users. That means that the significant average percentage improvement of the income of APs that was presented in Fig. 6 and Fig. 7 is a typical value of the performance of BestMax.

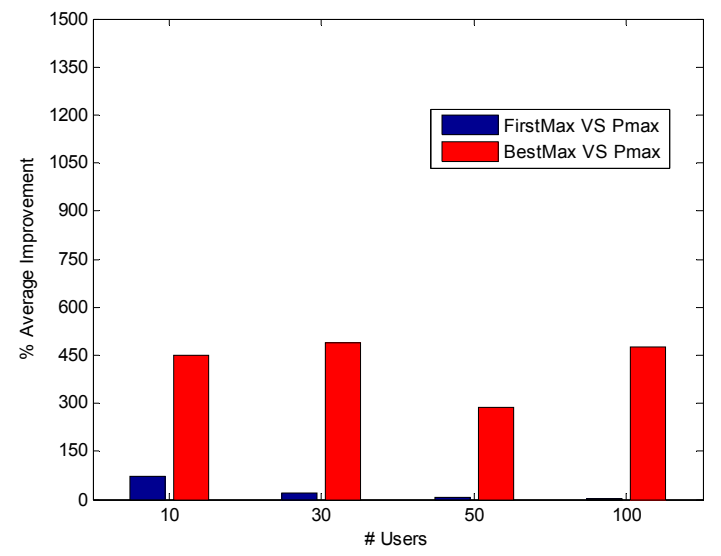

Fig. 6. Average percentage improvement of algorithms FirstMax and BestMax versus Pmax when the topology consists of two APs.

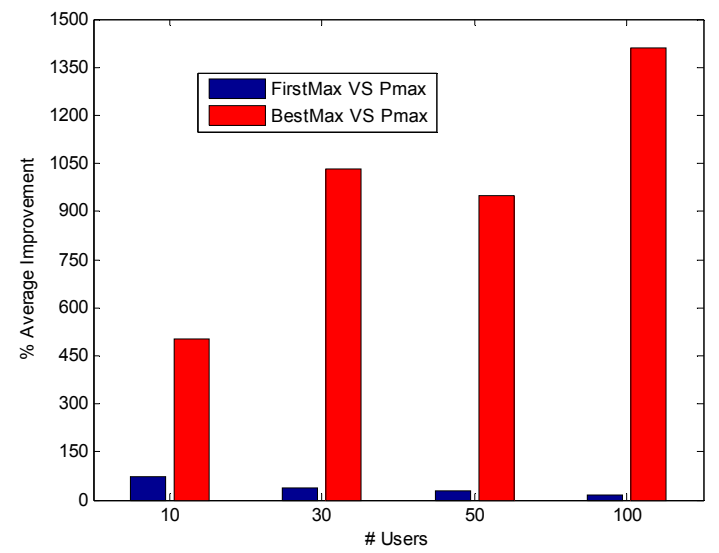

Fig. 7. Average percentage improvement of algorithms FirstMax and BestMax versus Pmax when the topology consists of three APs.

\section{CONCLUSIONS}

In this paper, we presented two algorithms that, taking as granted that all APs reduce together and to the same level their transmission's power, lead to as much fair distribution of incomes of APs as possible, i.e., to an optimization of the social fairness. Simulations show that the proposed algorithms (and especially BestMax) are far more profitable choices than the transmission of the APs at the maximum feasible power, as owners of APs can multiply many times their incomes by following them.

In our future work, we plan to explore mechanisms that apply bargaining or auctions among neighbor APs, where money or other in-kind compensation can be offered from an AP to its neighbors in exchange for reduction in their transmission power and/or some of their associated clients.

\section{REFERENCES}

[1] Mhatre, V. P.; Papagiannaki, K.; Baccelli, F., "Interference Mitigation Through Power Control in High Density 802.11 WLANs," Proc. IEEE INFOCOM'07, pp. 535-543, May 2007.

[2] Nie, N.; Comaniciu, C.; Agrawal, P., "A Game Theoretic Approach to Interference Management in Cognitive Networks," in Wireless Communications (The IMA Volumes of Mathematics and its Applications, P. Agrawal, D. M. Andrews, P. J. Fleming, G. Yin, and L. Zhang, eds.), Springer, November 2006.

[3] Berlemann, L.; Hiertz, G.R.; Walke, B.; Mangold, S., "Strategies for distributed QoS support in radio spectrum sharing," Proc. ICC'05, pp. 3271-3277, May 2005.

[4] Suris, J. E.; DaSilva, L. A.; Han, Z.; MacKenzie, A. B., "Cooperative Game Theory for Distributed Spectrum Sharing,", Proc. IEEE ICC'07, pp. 5282-5287, June 2007.

[5] Mitola, J., III; Maguire, G.Q., Jr., "Cognitive radio: making software radios more personal," IEEE Personal Communications, vol.6, no.4, pp.13-18, August 1999..

[6] Bahl, P.; Hajiaghayi, M.T.; Jain, K.; Mirrokni, S.V.; Qiu, L.; Saberi, A., "Cell Breathing in Wireless LANs: Algorithms and Evaluation," IEEE Transactions on Mobile Computing, vol. 6, no. 2, pp. 164-178, Feb. 2007.

[7] Lin Du; Biaham, J.; Cuthbert, L., "A bubble oscillation algorithm for distributed geographic load balancing in mobile networks," Proc. IEEE INFOCOM'04, pp. 330-338, March 2004.

[8] Balazinska, M; Castro, P., "Characterizing mobility and network usage in a corporate wireless local-area network," Proc. ACM MobiSys, 2003.

[9] Henderson, T.; Kotz, D.; Abyzov, I., "The Changing Usage of a Mature Campus-wide Wireless Network," Proc. ACM MobiCom 2004, pp. 187-201, Philadelphia, PA, USA, September 2004.

[10] Akella, A.; Judd, G.; Seshan, S.; Steenkiste, P., "SelfManagement in Chaotic Wireless Deployments," Proc. ACM MobiCom 2005, Cologne, Germany, September 2005. 